\title{
El uso de la información científica en la producción de tesis de posgrado de la Universidade Estadual Paulista, Marília
}

\author{
Ely Francina Tannuri de Oliveira \\ Helen de Castro Silva \\ Rodrigo Moreira Garcia \\ Universidade Estadual Paulista, Marília (Brasil)
}

\section{Resumen}

Este estudio tiene como objetivo investigar la utilización de la información científica y verificar el impacto del comportamiento de búsqueda y uso de los datos obtenidos anteriormente sobre las tesis elaboradas en los cuatro programas de posgrado de la UNESP, campus de Marília, en los últimos cinco años; identificar las fuentes citadas por estos alumnos en sus tesis de máster y obtener ayudas para caracterizar su comportamiento en cuanto al uso de la información en sus investigaciones y comparar los resultados obtenidos con la literatura sobre el tema, en especial en lo referente a las ciencias humanas y sociales. Para llevar a cabo el estudio se utilizó la técnica de análisis de citas de una muestra de 30 tesis defendidas en los últimos cinco años (2001-2005) en los cuatro programas de posgrado en dicha institución, a saber: Ciencias Sociales, Ciencia de la Información, Filosofía y Educación. Las referencias de cada tesis seleccionada fueron analizadas considerando el tipo de fuente, el soporte (impreso o electrónico), el promedio de citas por trabajo analizado y el idioma de las citas. A continuación se realizó un estudio comparativo con los indicadores que reflejan las fuentes más utilizadas en las diferentes áreas científicas. Los resultados demuestran que los alumnos de posgrado de la UNESP/Marília tienen predilección por las monografías (tesis o libros) y predominan de fuentes impresas en forma de libros; no obstante, la Universidad permite el acceso a una gran variedad de fuentes de información científica en línea. Los resultados confirman la tendencia de uso de la información, o sea, la utilización de fuentes impresas en forma de libros, para el área de ciencias humanas. Se observaron también algunas particularidades en las diferentes áreas, a pesar de que todas siguen patrones tradicionales de uso de las fuentes.

Palabras clave: Análisis de citas. Bibliometría. Comportamiento informativo. Estudio de uso. Ciencias sociales y humanidades. Bibliotecas universitarias. Desarrollo de colecciones.

Scire. $13: 2$ (jul.-dic. 2007) 129-137. ISSN 1135-3716. 


\section{Abstract}

The goal of this essay is to verify the impact of the behavior of the seeking and use of the information identified in prior researches in dissertations produced in the four post-graduation programs from UNESP, Marília Campus, in the last five years; to identify the sources reported by the post-graduand in their dissertations and to raise subsidies to characterize the behavior of the information use of the post-graduation students from UNESP/Marília and to compare the found results with the literature about this subject, in special those focused in human and social sciences. The accomplishment of this paper was based in analysis of the citations from a proportional sample of the dissertations produced in the last five years (2001-2005) in the four post-graduation programs from UNESP Marília: Social Science, Information Science, Philosophy and Education. Dissertations were selected by an aleatory sortition. The references of each selected paper were analyzed as for the kind of source that it refers to, support (printed or electronic), language and average of the reported sources by the dissertations. After that, a comparative study was done with the indicators that suggests, in different scientific areas, the most used sources in areas focused in the research. The results show that the post-graduand from UNESP/Marília prefer monographs (books, thesis and dissertations) as information source to theirs researches, and there is a predominance of printed sources, although the University offers the access to varied sources of on-line scientific information. The results confirm the tendency of the information use, that is, the use of printed sources as in a book, in human area. Although we could see some particularities in the different areas, all of them follow traditional standard of information use.

Key words: Citation analyisis. University libraries. Information behavior. Information use. Social sciences and humanities. Collection development.

\section{Introducción}

Los cursos de posgrado han promovido gran parte de las investigaciones y de la producción científica publicada en Brasil. Sabemos que para desarrollar investigaciones de calidad es fundamental el acceso a fuentes de información científica seguras y relevantes. El estudio del comportamiento de los individuos en la búsqueda y obtención de datos es importante para establecer la base de la ciencia de la información, pues, de acuerdo con Albrechtsen y Höjrland (1997), varios aspectos del área están subordinados a él.

Las acciones emprendidas por los individuos para obtener datos y solucionar sus necesidades de información pueden ser definidas como comportamiento de búsqueda y uso de la información o comportamiento informacional (Calva González, 1999). Este comportamiento varía de un área a otra dependiendo de la especialidad del investigador, tal como señala Calva González (1999, p. 14). 
Así, las diferentes áreas dan preferencia a distintos canales para divulgar sus resultados y llevar a cabo sus investigaciones. Esas variaciones, según Mueller (2005), dependen del prestigio o valor que cada área atribuye a los diversos canales de comunicación y divulgación.

El estudio del comportamiento informativo de los investigadores de diferentes áreas produce muchas veces resultados divergentes, y la comparación entre ellas es polémica. Así, los autores perfilan algunos indicadores que nos sirven de referencia.

Mueller (2005), por ejemplo, compara el uso de diferentes canales en las distintas las áreas. Para esta autora, en las ciencias experimentales las investigaciones se llevan a cabo habitualmente en equipo, se apoyan en paradigmas universalmente aceptados y se divulgan a través de artículos publicados en revistas científicas de circulación internacional y en lengua inglesa. Por el contrario, en las áreas clasificadas como ciencias sociales y humanidades, por lo general los estudios son realizados individualmente por los investigadores, quienes tienden a producir textos más extensos y que se publican principalmente como libros o capítulos de libros: "Nessas áreas podem conviver mais de uma abordagem teórica ou várias escolas de pensamento. Não há, também, uniformidade nos métodos adotados, havendo espaço para métodos quantitativos, semelhantes às ciências exatas, métodos qualitativos em suas várias versões e o uso de diversas combinações" (Mueller, 2005).

El comportamiento de los investigadores de las áreas de humanidades y ciencias sociales ha sido poco explotado en estudios sobre el tema (Calva González, 1999), especialmente en lo que se refiere a los brasileños.

Las investigaciones llevadas a cabo anteriormente sobre los alumnos de posgrado de la institución citada revelaron algunas cuestiones sobre el comportamiento informacional de este grupo (Omote, Prado y Carrara, 2003; Garcia y Silva, 2005; Silva, 2006). Uno de los resultados más destacables es que sus principales fuentes para obtener la información no son las bases de datos especializadas, incluso teniendo acceso gratuito a través de la Universidad.

A partir de estos datos se propuso la realización de la presente investigación, cuyos objetivos son $a$ ) verificar el impacto del comportamiento de búsqueda y uso de los datos obtenidos anteriormente sobre las tesis elaboradas en los cuatro programas de posgrado de la UNESP, campus de Marília, en los últimos cinco años; $b$ ) identificar las fuentes citadas por los alumnos de posgrado en sus tesis; $c$ ) obtener ayudas para caracterizar su comportamiento en cuanto al uso de la información; $d$ ) comparar los resultados obtenidos con la literatura sobre el tema, en especial en lo referente a las ciencias humanas y sociales.

La Universidad Estadual Paulista Julio de Mesquita Filho (UNESP), lugar donde se desarrolló la investigación aquí reflejada, es una de las mayores y más 
importantes universidades brasileñas y lleva a cabo una destacada actuación en enseñanza, investigación y extensión de sus actividades a la comunidad. Está financiada por el Gobierno del estado de São Paulo y ofrece enseñanza gratuita en 23 ciudades de dicho estado (1). La Facultad de Filosofía y Ciencia (FFC) está localizada en el interior del estado, en la ciudad de Marília (2). En el campus de la UNESP/Marília se ofrecen nueve cursos de graduación y cinco programas de posgrado sensu stricto, a saber: máster y doctorado en Educación y Ciencia de la Información y máster en Ciencias Sociales, Filosofía y Relaciones Internacionales. Todos los programas relacionados pertenecen a las áreas de humanidades y ciencias sociales aplicadas, de acuerdo con las tablas de áreas del conocimiento establecidas por el Consejo Nacional de Ciencia y Tecnología (CNPq), órgano ligado al Gobierno brasileño y responsable del apoyo al desarrollo de la investigación en Brasil (3).

\section{Metodología}

En el desarrollo de esta investigación se utilizó la técnica de análisis de citas para caracterizar la literatura utilizada por los alumnos de posgrado a la hora de realizar sus tesis. El análisis de citas es una técnica bibliométrica que estudia la relación entre los documentos, en general científicos, que se basan en investigaciones previas y son citados directa o indirectamente con el fin de comprobar datos o afirmaciones. El tipo de material citado se refiere a los documentos usados como referencia para elaborar las tesis analizadas.

El objeto de análisis de este estudio fueron las tesis defendidas en los últimos cinco años en los cuatro programas de posgrado con sede en la UNESP/Marília anteriormente citados (4).

Para la obtención de datos se tomó el conjunto de las tesis de maestría defendidas en los últimos cinco años en los cuatro programas de posgrado con sede en esta institución, lo que supone un total de 308 tesis. De estas, 187, o sea, el 61\%, son del programa de Educación; 53 (17\%), del de Ciencias Sociales; 43 (14\%), del de Filosofía, y 25 (8\%), del de Ciencia de la Información. A continuación, mediante sorteo, se obtuvo una muestra de 30 tesis por el método de muestreo estratificado y se consideró la proporción en la que estaban representadas en el conjunto de las 308 que componen el universo investigado. El resultado fue el siguiente: 18 tesis (el 61\% de las 30 que componen la muestra) eran del programa de Educación; 5 (17\%), del de Ciencias Sociales; 4 (14\%), del de Filosofía, y 3 (8\%), del de Ciencia de la Información.

Las fuentes citadas en cada una de las 30 tesis fueron analizadas y agrupadas en diferentes categorías: libros y capítulos de libros, tesis o tesis, artículos de revistas científicas o periódicos, anales de eventos y documentos oficiales, entre otras. Se comprobó el tipo de soporte de las fuentes citadas (impreso o electrónico) y el 
promedio de citas por disertación. Seguidamente se realizó un estudio comparativo con los indicadores que reflejan las fuentes más utilizadas en las diferentes áreas científicas.

\section{Presentación y discusión de los resultados}

En las 30 tesis estudiadas en la investigación se encontró un total de 2195 fuentes referenciadas, lo que da un promedio de 73 referencias por disertación. El primer aspecto analizado se refiere al soporte de los documentos utilizados por los alumnos de posgrado de la institución para la elaboración de sus tesis. Tal como se observa en la figura 1, de las 2195 referencias encontradas 1994 (90,8\%) eran documentos impresos y 201 (9,2\%) eran fuentes electrónicas, lo que demuestra una clara preferencia de los alumnos por el soporte impreso.

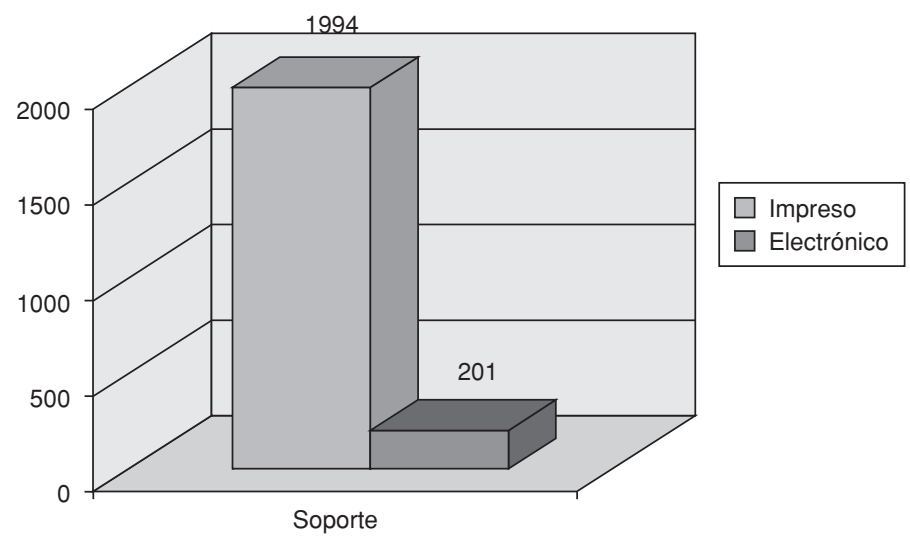

Figura 1. Soporte de los documentos citados.

El resultado no correspondió a lo previsto, ya que las investigaciones anteriormente mencionadas (Garcia y Silva, 2005; Silva, 2006) indicaban que los alumnos de posgrado utilizan Internet como una de sus principales fuentes de información para sus investigaciones, de modo que se esperaba obtener un alto índice de referencias de documentos electrónicos. Por tanto, debe hacerse un estudio más amplio para determinar con qué finalidad utilizaban Internet estos alumnos.

En relación con los tipos de fuentes citadas, se verificó que los más frecuentes son los libros y/o capítulos de libros, seguidos de los artículos de revistas científicas, tesis de máster o de doctorado, anales de eventos y documentos oficiales, como se ve en la figura 2.

Scire. 13 : 2 (jul.-dic. 2007) 129-137. ISSN 1135-3716. 


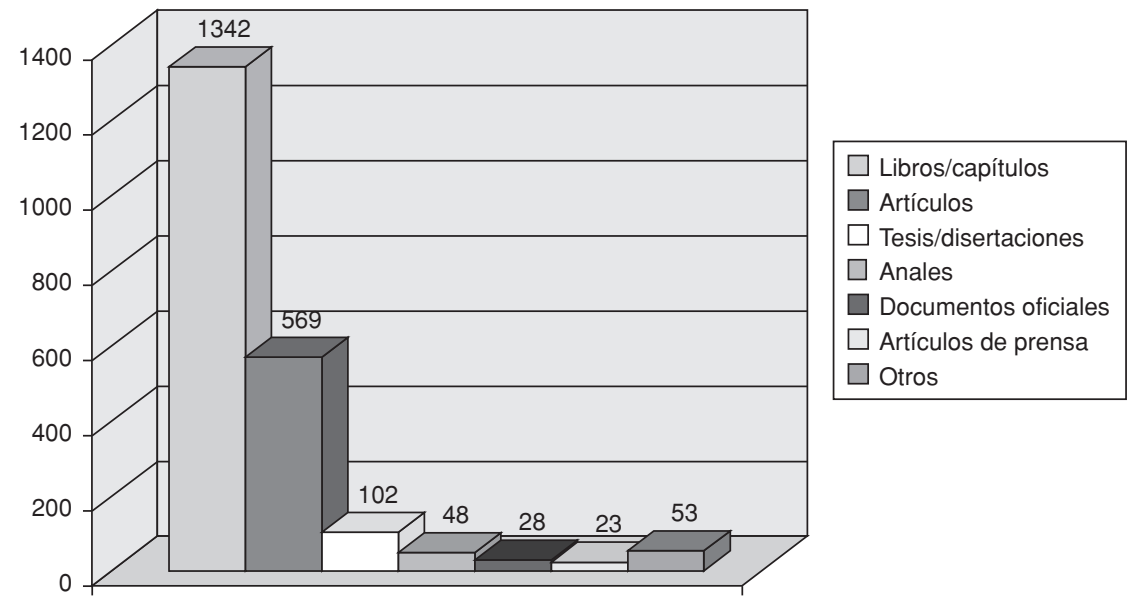

Figura 2. Tipos de fuentes de información citadas.

Estos resultados están de acuerdo con las tendencias internacionales de comportamiento informacional de las áreas de humanidades y ciencias sociales apuntadas por Calva González (1999) y más recientemente por Nederhof (2006). Según estos autores, las monografías (libros o tesis) son las principales fuentes de información para los investigadores de las áreas de humanidades y ciencias sociales, seguidas de las revistas científicas.

Los demás tipos de documentos encontrados en las tesis analizadas correspondían al $1 \%$ o menos del total de las citas y se incluyeron en la categoría "Otros": normas técnicas, con 14 citas; sitios de Internet y obras de referencia, con 11 cada uno; trabajo de fin de curso de graduación y/o especialización, con 10; documentos electrónicos, con 6, y letra de música, con 1.

Respecto al idioma de las fuentes citadas, se verificó que el $83 \%$ están en portugués, y solamente el 17\% en otras lenguas, lo que coincide con los patrones descritos por la literatura. Según Nederhof (2006), los resultados obtenidos para las áreas de ciencias sociales y humanidades no siempre son útiles para investigaciones de otros países. Algunas áreas muestran una fuerte inclinación hacia trabajos de ámbito regional o nacional, en los que no se suelen aplicar los resultados de otros contextos, y de ahí surge el predominio de documentos nacionales en las referencias de estudios de estas áreas.

Hasta aquí se han presentado los resultados generales que coinciden con los obtenidos en otros estudios sobre el tema. Según Mueller (2005), por ejemplo, tanto los investigadores del área de ciencias sociales aplicadas como los del área de cien-

Scire. $13: 2$ (jul.-dic. 2007) 129-137. ISSN 1135-3716. 
cias humanas muestran preferencia por los periódicos nacionales y a los libros, y publican con menor frecuencia en revistas científicas extranjeras, congresos nacionales y capítulos de libros.

El estudio demuestra que el comportamiento informacional es diferente en cada una de las áreas concretas incluidas en la investigación, lo que confirma la afirmación de Nederhof et ál. (1989) y Thompson (2002) (ápud Nederhof, 2006, p. 83), según los cuales "some of social sciences and humanities resemble natural and life sciences in publication and citation behavior, while others share characteristics with the tradicional profile of humanities scholarship". En vista de ello, se analizaron por separado los resultados obtenidos para cada una de las áreas, tal como se presentan a continuación.

El área de educación, por ejemplo, es la que presenta una mayor variedad de tipos de fuentes citadas, 12 en total. Las más citadas son libros y/o capítulos de libros, con 806 citas; artículos de periódicos, con 232; tesis de máster y/o de doctorado, con 68, y anales de eventos, con 38 . Se observa que esta área sigue la tendencia general de los tipos de fuentes más citadas expuesta anteriormente. Las tesis de esta área muestran un promedio de 68 fuentes referenciadas, índice un 7\% inferior a la media del conjunto de las tesis analizadas, que es de 73. Predominan las citas de fuentes en portugués, que corresponden al $87 \%$ del total (1057 citas). Esta es la segunda área en cuanto a preferencia por la utilización de material nacional.

El área de ciencias sociales también siguió la tendencia general de predilección en cuanto a tipos de fuentes citadas: libros y/o capítulos de libro, 292 citas; artículos de revistas científicas, 150; tesis de máster y/o de doctorado, 25, y anales de eventos, 21. Esta área fue la que presentó el mayor promedio de fuentes referenciadas, 100 citas por disertación, 27 (37\%) más que la media del conjunto de las tesis analizadas, que es de 73. En este caso se obtuvo la mayor incidencia de fuentes nacionales o traducidas al portugués, el $91 \%$ del total de las fuentes citadas.

El área de filosofía es la que presentó el mayor predominio de fuentes en formato impreso: el 98\% de las fuentes encontradas en las tesis analizadas eran documentos impresos. En las tesis de esta área había menor variedad de tipos de fuentes, solamente 4: libros y/o capítulos de libros, con 180 citas; artículos de revistas científicas, con 34; anales de eventos con 8, y tesis de máster y/o de doctorado, 2. En este caso se halló un menor número de referencias por disertación, un promedio de 56, 23\% menos que la media del conjunto de las tesis. Se obtuvieron 80 citas en lengua extranjera, que representan cerca del $36 \%$ del total de las referencias encontradas en las tesis del área.

Los resultados para el área de ciencia de la información demuestran que esta es la que más fuentes electrónicas utiliza en comparación con las demás áreas 
incluidas en el estudio: 78 (31\%) de las 253 referencias de las tesis analizadas son de documentos electrónicos. Los tipos de fuentes más citadas en este caso son artículos de revistas científicas, con 154 citas; libro, y/o capítulo, de libros, con 64; anales de eventos, con 11, y tesis de máster y/o de doctorado, con 7 . Esto demuestra que los alumnos de posgrado de esta área no tienen las mismas preferencias en cuanto al tipo de fuente que los de las otras tres áreas comprendidas en la investigación, en las que los libros y/o capítulos de libros son las fuentes más citadas. El área de ciencia de la información presentó 84 referencias por disertación, un promedio de 11 fuentes más que las demás áreas. También mostró el mayor índice de documentos extranjeros referenciados: 96, es decir, el $38 \%$ del total de las referencias encontradas en las tesis del área.

\section{Consideraciones finales}

En general, los resultados obtenidos en la investigación confirman la tendencia apuntada en la literatura en cuanto al uso de la información en las áreas de ciencias humanas y sociales en lo que se refiere a la preferencia por un determinado tipo de documentos (monografías, seguidas de artículos de revistas científicas), con predominio de las fuentes nacionales y en formato impreso. Aunque Tiratel (2000) llama la atención sobre el hecho de que la mayor parte de los estudios que demuestran la preferencia por el formato impreso en las áreas de ciencias sociales y humanidades fueron realizados antes de la difusión del uso de Internet, a través de este estudio se comprobó que dicha preferencia se mantiene todavía, al menos entre los alumnos de posgrado de la UNESP/Marília.

Otro aspecto que hay que tener en cuenta es la incidencia del comportamiento de búsqueda y uso de la información identificado en investigaciones anteriores (Silva, 2006; Garcia y Silva, 2005), que era uno de los objetivos de este estudio. Los resultados indicaron que no había una preferencia significativa por las herramientas de búsqueda en Internet a la hora de obtener de datos para el desarrollo de las tesis por parte de los alumnos de posgrado de la UNESP/Marília. Como los estudios previos indicaban que estos consideraban las herramientas de búsqueda en Internet como una de las principales fuentes de información para el desarrollo de sus investigaciones, se esperaba que las tesis presentasen un alto índice de citas de documentos electrónicos. Sin embargo, los resultados mostraron un predominio de citas de fuentes impresas y tradicionales, como libros, capítulos de libros, tesis de máster o de doctorado. Así pues, habría que investigar con qué finalidad usaron Internet estos alumnos.

A modo de sugerencia, debemos fomentar el uso de los recursos electrónicos disponibles tales como bases de datos referenciales y textuales de información científica de ámbito internacional entre los alumnos de las áreas de ciencias sociales y humanas. 


\section{Notas}

(1) Fuente: http://www.unesp.br/perfil/.

(2) Fuente: http://www.marilia.unesp.br/instituicao/apresentacao.

(3) Fuente: http://www.cnpq.br/areas/tabconhecimento/index.htm.

(4) El programa de Relaciones Internacionales se desarrolla en colaboración con otras universidades y no tiene su sede en Marília, de modo que no se incluyó en la presente investigación.

\section{Referencias}

Albrechttsen, H.; Hjörland, B. (1997). Information seeking and knowledge organization. // Knowledge Organization. 24:3 (1997) 136-144.

Calva González, Juan José (1999). El comportamiento en la búsqueda de información de los investigadores del área de humanidades y ciencias sociales. // Investigación Bibliotecológica. 13:27 (jul.-dic. 1999) 11-40.

Garcia, Rodrigo Moreira; Silva, Helen de Castro (2005). O comportamento do usuário final na recuperação temática da informação: um estudo com pós-graduandos da UNESP de Marília. // DataGramaZero. 6:3 (jun. 2005). http://www.dgz.org.br/jun05/F_I_art.htm (2005/08/11).

Mueller, Suzana P. M. (2005). A publicação da ciência: áreas científicas e seus canais preferenciais. // DataGramaZero. 6:1 (fev. 2005). http://www.dgz.org.br/fev05.htm (2005/08/11).

Nederhof, Anton J. (2006). Bibliometric monitoring of research performance in the social sciences and the humanities: a review. // Scientometrics. 66:1 (2006) 81-100.

Omote, S.; Prado, P. S. T.; Carrara, K (2003). O uso de fontes de referência na pesquisa bibliográfica por alunos de pós-graduação em educação. // V Simpósio em Filosofia e Ciência (Marília, 2003). Trabalho e conhecimento: desafios e responsabilidades das ciências: anais eletrônicos. Marília: UNESP Marília Publicações, 2003. CD-ROM.

Silva, Helen de Castro (2006). O comportamento do usuário final na busca e recuperação de informações: um estudo de caso com pós-graduandos em início de suas pesquisas. Relatório anual de atividades docentes, apresentado junto a Faculdade de Filosofia e Ciências da UNESP, Campus de Marília, 2006.

Altran, P.; Silva, H. C.; Oliveira, E. F. T. (2005). Fontes de informação citadas pelos alunos de pós-graduação da UNESP de Marília (1999-2004). // VI Simpósio de Filosofia e Ciências (Marília, 4-7 out. 2005). Marília: FFC UNESP Marília; Comissão Permanente de Publicações, 2005. CD-ROM.

Tiratel, Susana R. de (2000). Accessing information use by humanists and social scientists: a study at the Universidad de Buenos Aires, Argentina. // The Journal of Academic Librarianship. 26:56 (2000) 346-354. 